\title{
Survival model of a parallel system with dependent failures and time varying covariates
}

\begin{abstract}
In this paper, we extended a parallel system survival model based on the bivariate exponential to incorporate a time varying covariate. We calculated the bias, standard error and rmse of the parameter estimates of this model at different censoring levels using simulated data. We then compared the difference in the total error when a fixed covariate model was used instead of the true time varying covariate model. Following that, we studied three methods of constructing confidence intervals for such models and conclusions were drawn based on the results of the coverage probability study. Finally, the results obtained by fitting the diabetic retinopathy study data to the model were analysed.
\end{abstract}

Keyword: Bivariate,Covariates,Censoring,Jackknife 